\title{
Sophocarpine Protects Mice from ConA-Induced Hepatitis via Inhibition of the IFN-Gamma/STAT1 Pathway
}

\author{
Xiu-Xiu Sang ${ }^{1 \dagger}$, Rui-Lin Wang ${ }^{2 \dagger}$, Cong-En Zhang ${ }^{1+}$, Shi-Jing Liu' ${ }^{2}$, Hong-Hui Shen ${ }^{1}$, \\ Yu-Ming Guo ${ }^{1}$, Ya-Ming Zhang ${ }^{1}$, Ming Niu', Jia-Bo Wang ${ }^{1 *}$, Zhao-Fang Bai ${ }^{1 *}$ and \\ Xiao-He Xiao ${ }^{1 *}$ \\ ${ }^{1}$ China Military Institute of Chinese Medicine, 302 Military Hospital, Beijing, China, ${ }^{2}$ Integrative Medical Center, 302 Military \\ Hospital, Beijing, China
}

\section{OPEN ACCESS}

Edited by:

Annalisa Bruno,

University of Chieti-Pescara, Italy

Reviewed by:

Thomas Heinbockel,

Howard University, USA

Hai Li,

Shanghai Jiao Tong University, China

Yanqiong Zhang,

China Academy of Chinese Medical

Sciences, China

*Correspondence:

Zhao-Fang Bai

baizf2008@126.com

Xiao-He Xiao

pharmacy302@126.com

Jia-Bo Wang

wjb0128@126.com

†These authors have contributed equally to this work.

Specialty section: This article was submitted to Inflammation Pharmacology,

a section of the journal

Frontiers in Pharmacology

Received: 24 November 2016 Accepted: 06 March 2017

Published: 21 March 2017

Citation:

Sang $X-X$, Wang $R-L$, Zhang $C-E$,

Liu S-J, Shen H-H, Guo Y-M,

Zhang $Y-M$, Niu M, Wang J-B, Bai Z-F and Xiao X-H (2017) Sophocarpine

Protects Mice from ConA-Induced Hepatitis via Inhibition of the

IFN-Gamma/STAT1 Pathway.

Front. Pharmacol. 8:140.

doi: 10.3389/fphar.2017.00140
Sophocarpine is the major pharmacologically active compound of the traditional Chinese herbal medicine Radix Sophorae Subprostratae which has been used in treating hepatitis for years in China. It has been demonstrated that Sophocarpine exerts an activity in immune modulation and significantly decreases the production of inflammatory cytokines. However, the protective effects of Sophocarpine in T cell-dependent immune hepatitis remained unknown. The aim of this study was to determine the protective effects and pharmacological mechanisms of Sophocarpine on Concanavalin A (ConA)-induced hepatitis, an experimental model of $\mathrm{T}$ cell-mediated liver injury. BALB/C mice were pretreated with Sophocarpine or Bicyclol for five consecutive days. Thirty minutes after the final administration, the mice were injected with $15 \mathrm{mg} \cdot \mathrm{kg}^{-1}$ of ConA intravenously. The results indicated that pretreatment with Sophocarpine significantly ameliorated liver inflammation and injury as evidenced by both biochemical and histopathological observations. Moreover, in Sophocarpinepretreated mice, liver messenger RNA expression levels of chemokines and adhesion molecules, such as macrophage inflammatory protein-1 $\alpha$, CXC chemokine ligand 10, and Intercellular adhesion molecule-1, were markedly reduced. Further studies revealed that Sophocarpine significantly downregulated the expression of T-bet via inhibition of signal transducers and activators of transcription1 (STAT1) activation and overexpression of suppressor of cytokine signaling1, inhibiting the activation of Th1 cells and the expression of Interferon- $\gamma$ (IFN- $\gamma$ ). Altogether, these results suggest new opportunities to use Sophocarpine in the treatment of T cell-mediated liver disease. In summary, Sophocarpine could attenuate ConA-induced liver injury, and the protective effect of Sophocarpine was associated with its inhibition effect of pro-inflammatory cytokines, chemokines, and the IFN- $\gamma /$ STAT1 signaling pathway.

Keywords: Sophocarpine, Concanavalin A-induced hepatitis, interferon- $\gamma$, signal transducers and activators of transcription1, suppressor of cytokine signaling1

Abbreviations: ALT, alanine aminotransferase; AST, aspartate aminotransferase; ConA, Concanavalin A; CXCL10, chemokine ligand 10; ICAM-1, intercellular adhesion molecule-1; IFN- $\gamma$, interferon- $\gamma$; IL, interleukin; LPS, lipopolysaccharide; MIP-1 $\alpha$, macrophage inflammatory protein-1 $\alpha$; SOCS1, suppressor of cytokine signaling 1; STAT1, signal transducers and activators of transcription1; TBIL, total bilirubin; TGF- $\beta 1$, transforming growth factor- $\beta 1$; TNF- $\alpha$, tumor necrosis factor- $\alpha$ 


\section{INTRODUCTION}

Hepatitis, which is caused by alcohol drinking, certain drugs, viral infection, or autoimmunity, is a serious threat to human health (Zheng et al., 2016). ConA-induced autoimmune hepatitis has been widely used in studies of immune hepatitis treatment in humans which has been considered a well-established experimental model for immune-mediated liver injury. CD4 T cells were essential to mediate adaptive immunity in response to a variety of pathogens. Intravenous application of the ConA in mice results in the activation of naive CD4 T cells and then subsequent differentiation into $\mathrm{T}$ helper cells, including Th1 (secrete IFN- $\gamma$ ), Th2 [secrete Interleukin-4 (IL-4)], Th17 cells (secrete IL-17), and Treg cells (Schumann et al., 2003; Siebler et al., 2003; Stummvoll et al., 2008; Manns et al., 2015). In the pathogenesis of ConA injection, IFN- $\gamma$ was reported to be critically involved in liver injury because in the mice deficient in IFN- $\gamma$ or treated with IFN- $\gamma$-neutralizing monoclonal antibody its disease progress was significantly reduced (Miyagi et al., 2004). IFN- $\gamma$ was also reported to activate STAT1, inducing T-bet expression, which is essential for Th1 cell differentiation (Shinohara et al., 2005; Zhu et al., 2010). SOCS1 is a negative regulator of STAT1 activation. It has been reported that overexpression of SOCS1 prevents ConA-induced liver injury by suppressing STAT1 activation (Ogata et al., 2006).

Sophocarpine exhibits many pharmacological activities and is a major matrine-type alkaloid present in the traditional Chinese herb, Radix Sophorae Subprostratae. Sophocarpine has been demonstrated to alleviate pain effectively in thermally and chemically induced mouse models (Gao et al., 2009), and it also inhibits LPS-mediated NF- $\kappa$ B activation in RAW 264.7 cells (Gao et al., 2012). Moreover, Sophocarpine can decrease serum levels of aminotransferase and TBIL and alleviate liver fibrosis by inhibiting the TLR4 pathway (Qian et al., 2014). It also exerts an activity in immune modulation (Zhang et al., 2007). Despite the evidence presented by these experimental studies, little is known regarding the effect of Sophocarpine on ConA-induced immunity hepatitis.

The aim of this study was to determine whether Sophocarpine could inhibit immune-mediated liver disease in T cell-dependent hepatitis mouse model. In addition, we aimed to define potential mechanisms by which Sophocarpine exerts its hepatoprotective role in ConA-induced liver injury.

\section{MATERIALS AND METHODS}

\section{Mice}

Male BALB/C mice, weighing approximately $18 \pm 22 \mathrm{~g}$, were acquired from SPF Animals Biotechnology Co., Ltd. (Beijing, China) (Certification number SCXK-JING 2011-0004). The mice were housed in the Institutional Laboratory Animal Care center at 302 Military Hospital. All of the animals were maintained according to the National Institutes of Health Guidelines for Animal Care and the Guidelines of Scripps. The protocol was approved by the Committee on the Ethics of Animal Experiments of the 302 Military Hospital (approval ID: IACUC-2016-008).
Efforts were made to minimize animal suffering and to reduce the number of animals used in the experiments. The animals were randomized into Control, Model, Bicyclol (100 mg. $\left.\mathrm{kg}^{-1}\right)$, Sophocarpine (30 and $60 \mathrm{mg} \cdot \mathrm{kg}^{-1}$ ) groups. The drugs were dissolved in saline and orally administered for five consecutive days. All of the mice in the control group were intragastrically administered with an equivalent volume of free saline. Thirty minutes after the final administration of medication, the mice were injected with $15 \mathrm{mg} \cdot \mathrm{kg}^{-1}$ of ConA intravenously by tail except for the normal group. The mice were sacrificed $8 \mathrm{~h}$ after injection. Blood samples were obtained, and the liver was collected simultaneously.

\section{Blood Chemistry Measurement}

The serum levels of ALT, AST, and TBIL were determined using the detection kits provided by Nanjing Jiancheng Bioengineering Institute (Nanjing, China). All procedures were performed according to the manufacturer's instructions. Serum was diluted fivefold for the detection of aminotransferase.

\section{Liver Histology, TUNEL Staining, and Immunohistochemistry}

Livers were fixed with $4 \%$ paraformaldehyde and embedded in paraffin. Next, they were cut into $5 \mu \mathrm{m}$-thick sections and the sections were stained with hematoxylin and eosin (H\&E) or a TUNEL kit was used (Promega, USA). Immunohistochemistry was performed by liver sections and stained with ICAM-1 antibody. The levels of tissue damage and immune complexes were examined using light microscopy.

\section{Analysis of Plasma Cytokines}

Plasma concentrations of TNF- $\alpha$, IFN- $\gamma$, IL-10, and TGF- $\beta 1$ were determined using ELISA kits (MultiSciences Biotech Co., Ltd., Hangzhou, China) according to the manufacturer's instructions. All indexes were measured $8 \mathrm{~h}$ after ConA injection.

\section{Quantitative PCR}

Total RNA was isolated from liver tissues by Trizol (Life Sciences) and first-strand cDNA synthesis was performed in $20 \mu \mathrm{l}$ of solution using the Revert kit (Thermo-Fisher). The mRNA expression level was quantified using real-time PCR (Applied Biosystems 7500 Real-Time PCR System) and a SYBR Green PCR Mix kit (Life Sciences). The primers sequences were as follows: GAPDH, 5'-GTCCTCAGTGTAGCCCAAGAT-3' and $5^{\prime}$-CAATGTGTCCGTCGTGGATCT-3'; T-bet, 5'-TGCC CGAACTACAGTCACGAAC-3' and 5'-AGTGACCTCGCCTG GTGAAATG-3'; GATA3, 5'-GGAGGACTTCCCCAAGAGCA$3^{\prime}$ and $5^{\prime}$-CATGCTGGAAGGGTGGTGA-3'; SOCS1, 5' -TCCGA TTACCGGCGCATCACG- $3^{\prime}$ and $5^{\prime}$-CTCCAGCAGCTCGAAA AGGCA-3'; MIP-1 $\alpha, \quad 5^{\prime}$-CACCCTCTGTCACCTGCTCAA-3 ${ }^{\prime}$ and $5^{\prime}$-ATGGCGCTGAGAAGACTTGGT-3'; ICAM-1, 5' -CCAT CACCGTGTATTCGTTTCC- $3^{\prime}$ and $5^{\prime}$-CTGGCGGCTCAGTAT CTCCTC-3'; CXCL10, 5' -TCCAGTTAAGGAGCCCTTTTAGA CC-3' and 5'-TGAAATCATCCCTGCGAGCCTAT-3'. The CT values were then converted to fold induction over normal at time zero (control) using the $2^{-\Delta \Delta \mathrm{CT}}$ formula. 


\section{Isolation of Lymphocytes and Flow Cytometry}

$1^{*} 10^{6}$ splenocytes from the different groups of experimental mice were stimulated for $6 \mathrm{~h}$ with PMA (100 $\mathrm{ng} / \mu \mathrm{l})$, Ionomycin $(1 \mu \mathrm{g} / \mu \mathrm{l})$, and Monensin $(1 \mu \mathrm{g} / \mu \mathrm{l})$ and stained with the Percy/cy5.5-conjugated anti-CD3 (Dakewe Biotech Co., Ltd.) and PE/cy-7-conjugated anti-CD8 (Dakewe Biotech Co., Ltd.); fluorescence antibodies specific for surface antigens were used according to the manufacturer's instructions. For intracellular cytokine staining, the cells were fixed, permeabilized, and stained with PE-conjugated anti-IL-4 (BD Biotech Co., Ltd.) and FITCconjugated anti-IFN- $\gamma$ (BD Biotech Co., Ltd.). The stained cells were analyzed using a flow cytometer (BD Canto II), and the data were analyzed using FlowJo 7.6.1.

\section{Western Blotting Analyses}

Liver tissue was lysed on ice with RIPA buffer (G2002) containing a protease inhibitor mixture. After centrifugation, the samples were incubated for $10 \mathrm{~min}$ at $4^{\circ} \mathrm{C}$ to separate any debris. The membrane was incubated with antibodies against $\beta$-actin, T-bet, STAT1, and phospho-STAT1 (Tyr705) in TBS/T containing $5 \%$ non-fat milk overnight at $4^{\circ} \mathrm{C}$. After incubation with the appropriate peroxidase-conjugated secondary antibody, the membrane was washed in TBST for $5 \mathrm{~min}$.

\section{Statistical Analysis}

All results are expressed as the mean $\pm \mathrm{SD}$ and analyzed using SPSS software (version 20.0; SPSS, Inc., Chicago, IL, USA). Differences were considered to be significant when $p<0.05$ and highly significant when $p<0.01$.

\section{RESULTS}

\section{Effects of Sophocarpine on Serum in ConA-Induced Acute Hepatitis}

To examine the effect of Sophocarpine on ConA-induced acute hepatitis, mice were pretreated with Sophocarpine (30 or $60 \mathrm{mg} \cdot \mathrm{kg}^{-1}$ ) for five consecutive days. Bicyclol, as a novel derivative of Bifendate (Yuan et al., 2016) which has been confirmed to decrease the levels of serum ALT served as a positive parallel control, and control mice received saline accordingly. Thirty minutes after the final dose of medication, all the mice were injected with $15 \mathrm{mg} \cdot \mathrm{kg}^{-1}$ of body weight of ConA via the tail vein except the normal group. Eight hours later, plasma was obtained and serum levels of ALT, AST, and TBIL were measured. Compared with the normal group, the ALT (Control: $8.20 \pm 1.24$, Model: $957.92 \pm 76.46$ ), AST (Control: $5.64 \pm 1.45$, Model: $344.98 \pm 58.50$ ), and TBIL (Control: $1.26 \pm 0.66$, Model: $9.99 \pm 2.67$ ) in the ConA group were significantly increased. Administration of Bicyclol and Sophocarpine inhibited the elevation of ALT [Bicyclol: $628.12 \pm 205.94$, Sophocarpine $\left(30 \mathrm{mg} \cdot \mathrm{kg}^{-1}\right): 398.02 \pm 178.35$, Sophocarpine $\left(60 \mathrm{mg} \cdot \mathrm{kg}^{-1}\right): 171.25 \pm 113.68$ ], AST [Bicyclol: $219.81 \pm 83.58$, Sophocarpine $\left(30 \mathrm{mg} \cdot \mathrm{kg}^{-1}\right): 157.69 \pm 59.55$, Sophocarpine $\left(60 \mathrm{mg} \cdot \mathrm{kg}^{-1}\right): 84.46 \pm 25.28$ ], and TBIL [Bicyclol: $5.08 \pm 1.13$, Sophocarpine $\left(30 \mathrm{mg} \cdot \mathrm{kg}^{-1}\right): 5.05 \pm 1.42$, Sophocarpine $\left.\left(60 \mathrm{mg} \cdot \mathrm{kg}^{-1}\right): 3.21 \pm 1.25\right]$ compared with the ConA group (Figures 1A-C). Moreover, Sophocarpine inhibited ConA-induced aminotransferase release and TBIL increase in a dose-dependent manner. Taken together, these data significantly show the protective effect of Sophocarpine in ConA-induced hepatitis.

\section{Sophocarpine Attenuated Immunological Liver Injury in Mice}

Histopathological examinations of livers showed that, compared with the normal group, the model group revealed severe injury characterized by extensive inflammatory infiltration around the central veins (Figures 2A,B). In contrast, mice pretreated with Bicyclol and Sophocarpine demonstrated much less inflammatory infiltration (Figures 2C-E). In the Sophocarpine-pretreated group, only a few hepatocytes exhibited TUNEL-positive nuclei compared with the model group (Figures 2F-K) [Control: $0.57 \pm 0.26$, Model: $13.53 \pm 3.86$, Bicyclol: $6.23 \pm 1.22$, Sophocarpine $\left(30 \mathrm{mg} \cdot \mathrm{kg}^{-1}\right): 8.43 \pm 1.91$,
A

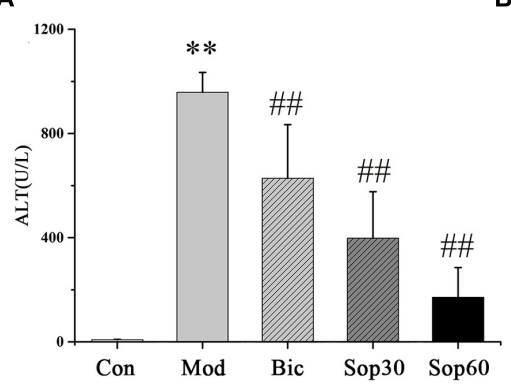

B

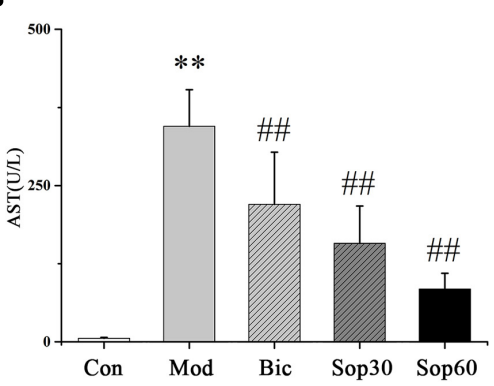

c

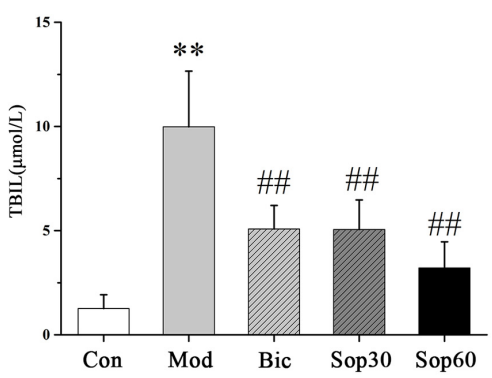

FIGURE 1 | Effects of Sophocarpine on serum in ConA-induced acute hepatitis. Female BALB/c mice were pretreated with Sophocaripine (30, $60 \mathrm{mg} \cdot \mathrm{kg}^{-1}$ ) and Bicyclol at 30 min before ConA injection. Serum transaminase ALT (A) and AST (B) TBIL (C) levels were determined $8 \mathrm{~h}$ after ConA injection. Data is expressed as mean $\pm \mathrm{SD}\left(n=12 ;{ }^{*} p<0.05,{ }^{* *} p<0.01\right.$ compared with the control group, ${ }^{\#} p<0.05,{ }^{\# \#} p<0.01$ compared with the model group, respectively). Saline group (Con group), ConA group (Mod group), Bicyclol group (Bic group), $30 \mathrm{mg} \cdot \mathrm{kg}^{-1}$ of Sophocarpine group (Sop30 group), and $60 \mathrm{mg} \cdot \mathrm{kg}^{-1}$ of Sophocarpine group (Sop60 group). 

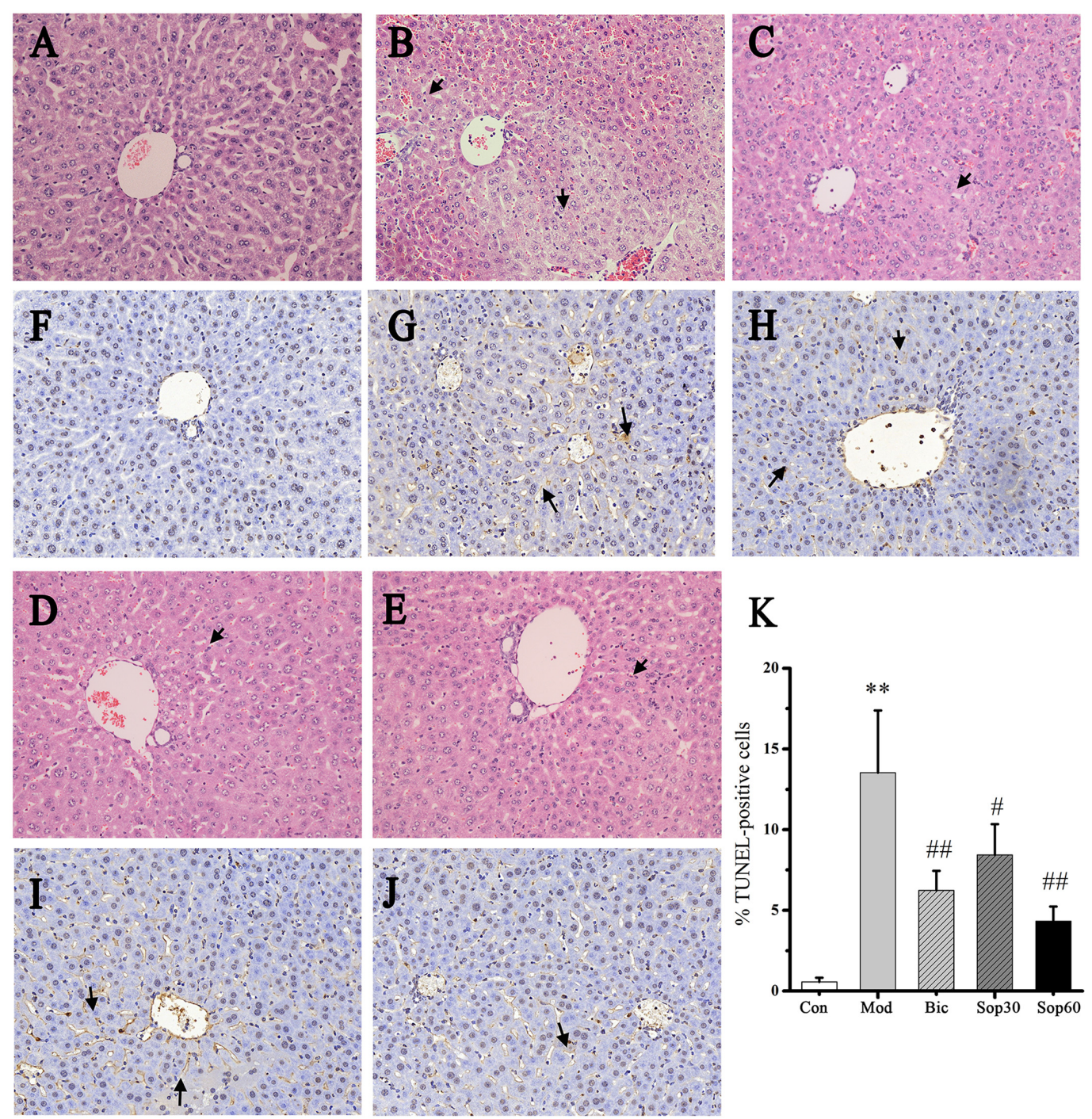

FIGURE 2 | Sophocarpine attenuated immunological liver injury in mice. Mice were treated with different doses of Sophocarpine. Representative photographs of six animals show the effect of Sophocarpine, which was confirmed by hematoxylin and eosin (H\&E) and TUNEL Staining. HE staining for (A) control mice, (B) model mice, (C) Bicyclol group, (D) $30 \mathrm{mg} \cdot \mathrm{kg}^{-1}$ of Sophocarpine group, (E) $60 \mathrm{mg} \cdot \mathrm{kg}^{-1}$ of Sophocarpine group. TUNEL staining for (F) control mice, (G) model mice, (H) Bicyclol group, (I) $30 \mathrm{mg} \cdot \mathrm{kg}^{-1}$ of Sophocarpine group, (J) $60 \mathrm{mg} \cdot \mathrm{kg}^{-1}$ of Sophocarpine group, and (K) Percentage of TUNEL-positive cells $(* * p<0.01$ compared with the control group, ${ }^{\#} p<0.05,{ }^{\# \#} p<0.01$ compared with the model group, respectively) (H\&E, magnification $\times 200$; TUNEL, magnification $\times 200$ ).

Sophocarpine $\left.\left(60 \mathrm{mg} \cdot \mathrm{kg}^{-1}\right): 4.33 \pm 0.90\right]$, indicating markedly reduced apoptosis in Sophocarpine-pretreated mice. Altogether, these results showed that Sophocarpine dose-dependently inhibited ConA-induced liver inflammation and cell death.

\section{Sophocarpine Inhibited the Expression of Chemokines and Adhesion Molecules in Mice with ConA-Induced Hepatitis}

In ConA-induced hepatitis, the expression of chemokines and adhesion molecules were demonstrated to play important roles in recruiting lymphocytes into the liver to aggravate liver injury, such as MIP-1 $\alpha$, CXCL10, and ICAM-1 (Ajuebor et al., 2004; Kawasuji et al., 2006; Sahin et al., 2013).
Thus, further studies were performed to examine the changes induced by these factors in the mice liver. Liver tissues were obtained $8 \mathrm{~h}$ after ConA administration, and mRNA expression levels of MIP-1 $\alpha$, CXCL10, and ICAM-1 were measured. As shown in Figure 3, the mRNA levels of MIP-1 $\alpha$ [Control: $1.00 \pm 0.72$, Model: $41.47 \pm 17.03$, Sophocarpine $\left(30 \mathrm{mg} \cdot \mathrm{kg}^{-1}\right)$ : $16.80 \pm 8.45$, Sophocarpine $\left.\left(60 \mathrm{mg} \cdot \mathrm{kg}^{-1}\right): 3.32 \pm 0.84\right]$, CXCL10 [Control: $1.00 \pm 0.29$, Model: $123.72 \pm 22.81$, Sophocarpine $\left(30 \mathrm{mg} \cdot \mathrm{kg}^{-1}\right): 57.05 \pm 16.03$, Sophocarpine $\left(60 \mathrm{mg} \cdot \mathrm{kg}^{-1}\right)$ : $18.56 \pm 4.59$ ], and ICAM-1[Control: $1.00 \pm 0.19$, Model: $43.44 \pm 12.33$, Sophocarpine $\left(30 \mathrm{mg} \cdot \mathrm{kg}^{-1}\right): 20.69 \pm 6.41$, Sophocarpine $\left.\left(60 \mathrm{mg} \cdot \mathrm{kg}^{-1}\right): 3.35 \pm 0.50\right]$ were dramatically reduced by pretreatment with Sophocarpine. Taken together, these results indicate that pretreatment with Sophocarpine 

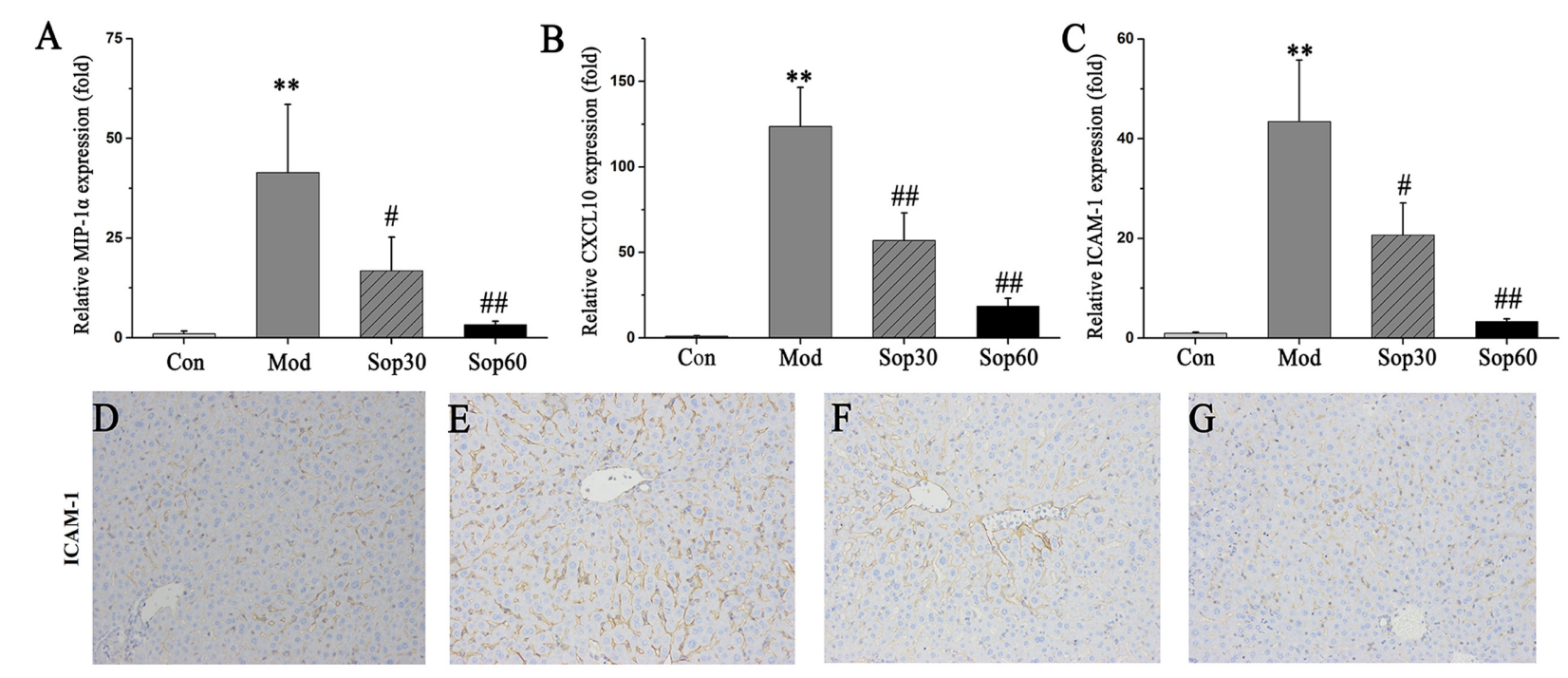

FIGURE 3 | Sophocarpine inhibited chemokine and adhesion molecule expression in the liver. BALB/C mice received Sophocarpine pretreatment and ConA injection, $8 \mathrm{~h}$ later liver tissues were obtained for quantitatively PCR and immunohistochemistry for ICAM-1. The mRNA expression levels of MIP-1 $\alpha$ (A),

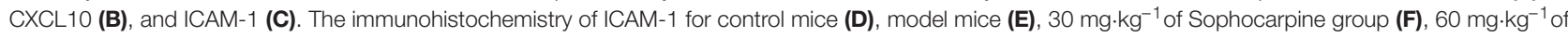
Sophocarpine group (G) $\left(n=6\right.$ each group). ${ }^{* *} p<0.01$ compared with the control group, ${ }^{\#} p<0.05$, ${ }^{\# \#} p<0.01$ compared with the model group.

significantly reduced ConA-induced intrahepatic inflammatory cell infiltration.

\section{Sophocarpine Inhibited the Production of IFN- $\gamma$ in Mice with ConA-Induced Hepatitis}

It was previously reported that following ConA administration, immune cells were activated. Consequently, various cytokines that aggravated the liver injury were released (Miyagi et al., 2004; Wang et al., 2012). To examine the protective mechanisms of Sophocarpine against liver injury, the effects of Sophocarpine on the serum levels of IFN- $\gamma$, TNF- $\alpha$, IL-10, and TGF- $\beta 1$ were measured using ELISA. The percentage of $\mathrm{CD} 4^{+} \mathrm{IFN}-\gamma^{+}$ and $\mathrm{CD} 4^{+} \mathrm{IL}-4^{+}$in Sophocarpine-pretreated mice splenocytes were examined using flow cytometry. These results showed that Sophocarpine inhibited the production of IFN- $\gamma$ [Control: $59.52 \pm 23.83$, Model: $3248.00 \pm 141.02$, Sophocarpine $\left(30 \mathrm{mg} \cdot \mathrm{kg}^{-1}\right): 2831.00 \pm 160.28$, Sophocarpine $\left(60 \mathrm{mg} \cdot \mathrm{kg}^{-1}\right)$ : $2030.00 \pm 242.52$ ] and TNF- $\alpha$ [Control: $245.52 \pm 73.64$, Model: $998.35 \pm 266.91$, Sophocarpine $\left(30 \mathrm{mg} \cdot \mathrm{kg}^{-1}\right): 1009.78 \pm 279.96$, Sophocarpine $\left.\left(60 \mathrm{mg} \cdot \mathrm{kg}^{-1}\right): 540.99 \pm 116.05\right]$ in serum and decreased the percentage of $\mathrm{CD}^{+}{ }^{+} \mathrm{IFN}-\gamma^{+}$[Control: $1.47 \pm 0.47$, Model: $2.89 \pm 0.10$, Sophocarpine $\left(30 \mathrm{mg} \cdot \mathrm{kg}^{-1}\right)$ : $1.87 \pm 0.45$, Sophocarpine $\left.\left(60 \mathrm{mg} \cdot \mathrm{kg}^{-1}\right): 1.41 \pm 0.77\right]$ in splenocytes compared with the model group (Figure 4), but there was no significant difference among the treatment groups in regards to the percentage of $\mathrm{CD} 4^{+} \mathrm{IL}-4^{+}$and the expression of IL-10 and TGF- $\beta 1$. Therefore, Sophocarpine effectively suppressed the production of IFN- $\gamma$ and TNF- $\alpha$ and reduced the liver damage caused by these inflammatory cytokines.

\section{Sophocarpine Downregulated the Expression of T-bet via Inhibition of STAT1 Activation and Overexpression SOCS1}

It has been previously shown that ConA-mediated liver damage can be prevented by blocking antibodies against IFN$\gamma$ rather than TNF, suggesting that IFN- $\gamma$ is an essential key regulator in ConA-induced liver injury (Tagawa et al., 1997). The transcription factor STAT1, which is associated with the IFN- $\gamma$ signaling pathway, is crucial for ConA-induced hepatitis. Activation of STAT1 by IFN- $\gamma$ is significant for the induction of T-bet during Th1 cells differentiation (Siebler et al., 2003; Jaruga et al., 2004). Therefore, we investigated the effect of Sophocarpine on the activation of STAT1 and the expression of T-bet in ConA-induced hepatitis. In the present study, Sophocarpine effectively inhibited STAT1 synthesis and activation as well as the expression of T-bet (Figure 5). SOCS1 has been shown to suppress inflammation and is indispensable for the negative regulation of STAT1 (Hashimoto et al., 2009). Therefore, gene expression analysis of the SOCS1 protein in the mouse livers was performed. The results indicated that Sophocarpine downregulated the expression of T-bet [Control: $27.44 \pm 11.55$, Model: $67.74 \pm 10.58$, Sophocarpine $\left(30 \mathrm{mg} \cdot \mathrm{kg}^{-1}\right): 36.81 \pm 4.51$, Sophocarpine $\left(60 \mathrm{mg} \cdot \mathrm{kg}^{-1}\right): 27.99 \pm 5.03$ ] via inhibition of STAT1 activation [Control: $24.22 \pm 14.78$, Model: $205.49 \pm 21.08$, Sophocarpine $\left(30 \mathrm{mg} \cdot \mathrm{kg}^{-1}\right): 75.72 \pm 16.64$, Sophocarpine $\left.\left(60 \mathrm{mg} \cdot \mathrm{kg}^{-1}\right): 8.21 \pm 3.34\right]$ and overexpression of SOCS1 [Control: $1.00 \pm 0.58$, Model: $1.76 \pm 0.31$, Sophocarpine $\left(30 \mathrm{mg} \cdot \mathrm{kg}^{-1}\right): 2.21 \pm 0.12$, Sophocarpine $\left(60 \mathrm{mg} \cdot \mathrm{kg}^{-1}\right)$ : $3.83 \pm 0.69]$, suggesting that Sophocarpine decreased the release of IFN- $\gamma$ by inhibiting the activation of the IFN- $\gamma /$ STAT1 signaling pathway and Th1 differentiation. 
A

D
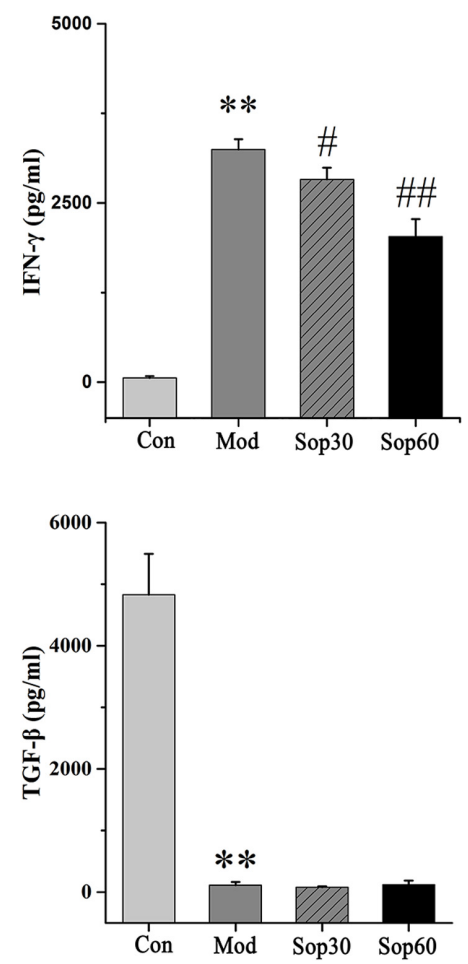

B

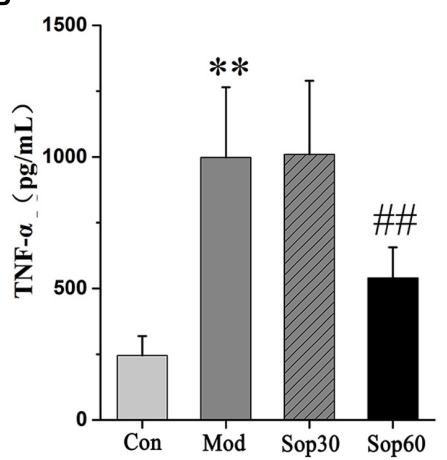

E

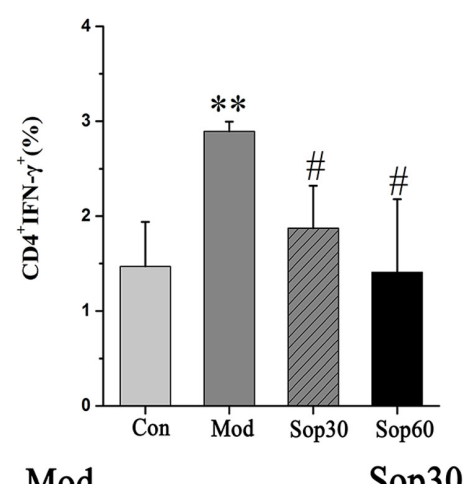

C

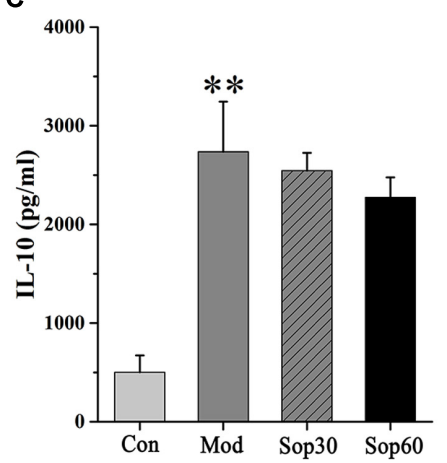

$\mathbf{F}$

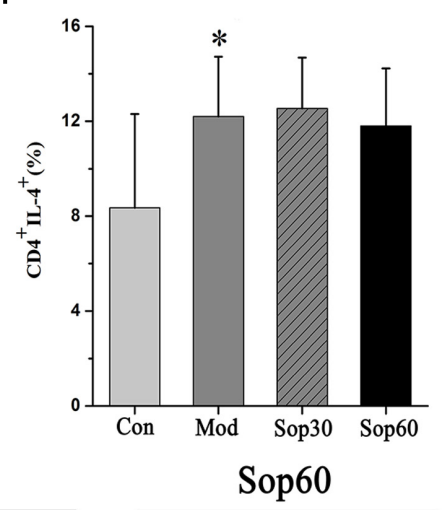

G Con
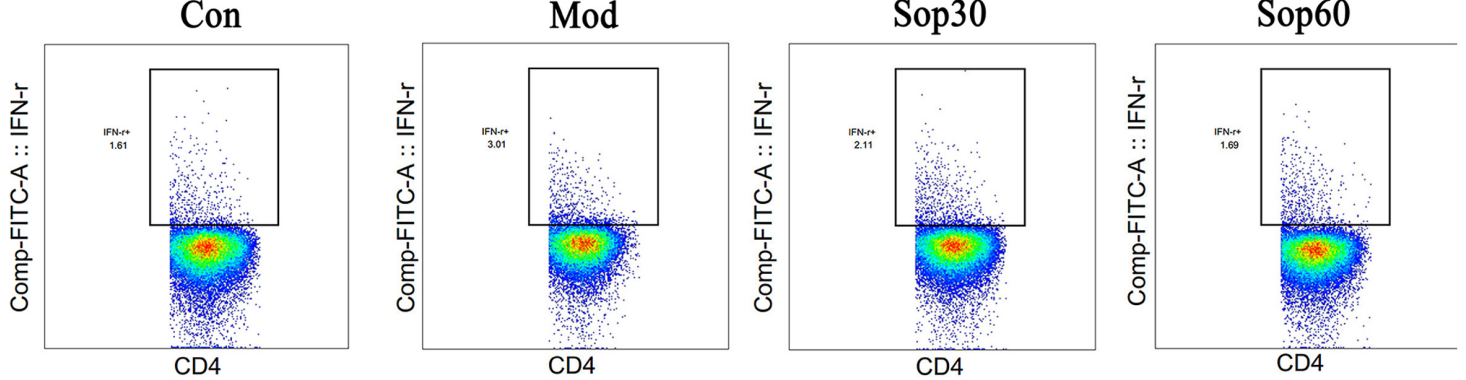

H
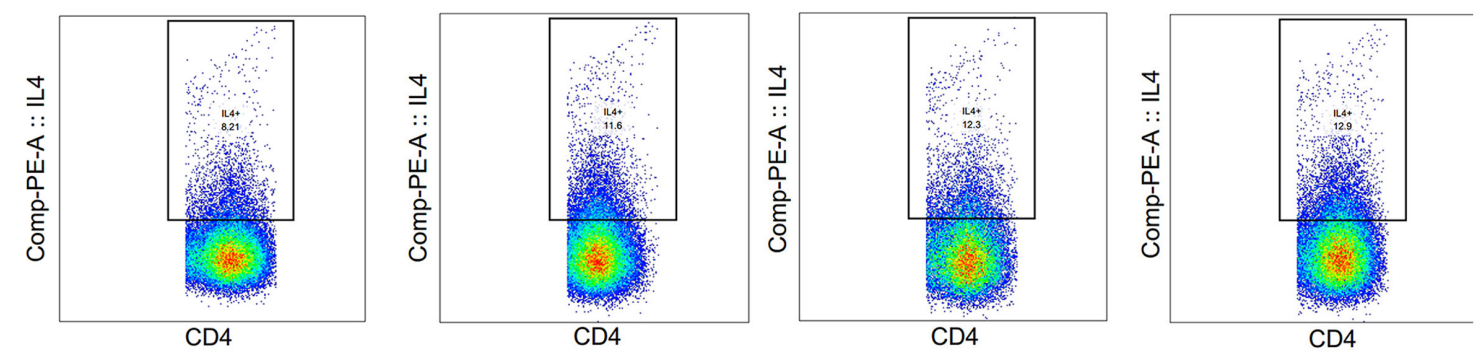

FIGURE 4 | Sophocarpine inhibited production of IFN- $\gamma$ in ConA-induced hepatitis mice. Blood samples were collected at $8 \mathrm{~h}$ after ConA injection. Serum levels of cytokines were detected by ELISA. Influences of Sophocaripine on inflammatory cells were measured by Flow Cytometric. Data is expressed as mean \pm SD. Serum ELISA for (A) IFN- $\gamma$, (B) TNF- $\alpha$, (C) IL-10, (D) TGF- $\beta 1$. Flow cytometry for (E) CD4 $4^{+}$IFN- $\gamma^{+}$, and (F) CD4 $4^{+} \mathrm{IL}-4^{+}$. Representative photos of each group for (G) $\mathrm{CD} 44^{+} \mathrm{IFN}-\gamma^{+}$, (H) CD4+IL-4+. $\left(n=6\right.$; ${ }^{*} p<0.05,{ }^{* *} p<0.01$ compared with the control group, ${ }^{\#} p<0.05,{ }^{\# \#} p<0.01$ compared with the model group).

\section{DISCUSSION}

An increasing number of traditional Chinese herbal medicines have been reported to serve as potential therapeutic protection against liver disease (Ding et al., 2012; Hu et al., 2013).
Sophocarpine is a quinolizidine alkaloid extracted from Radix Sophorae Subprostratae a traditional Chinese herb that has been widely used in the treatment of liver diseases for years in China. In previous studies, Sophocarpine has been reported to significantly decrease the production of inflammatory cytokines 
A

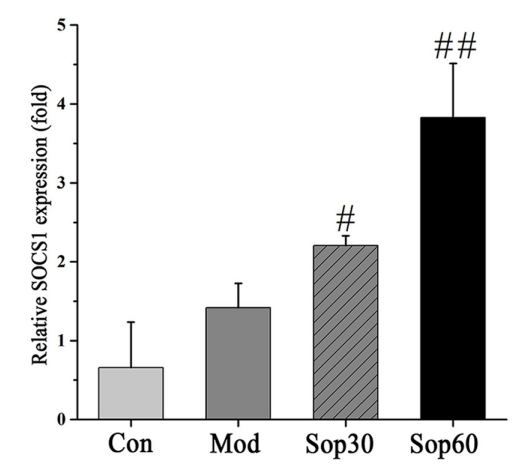

D

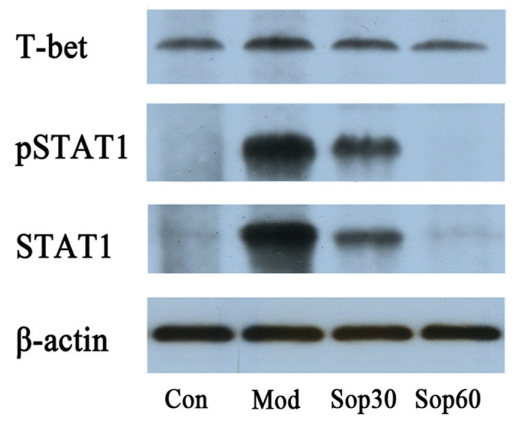

B

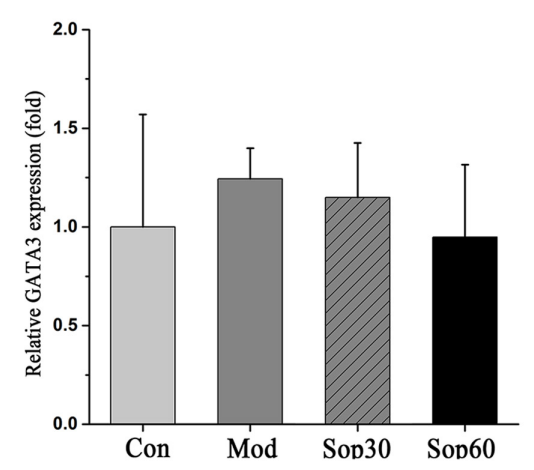

C

E

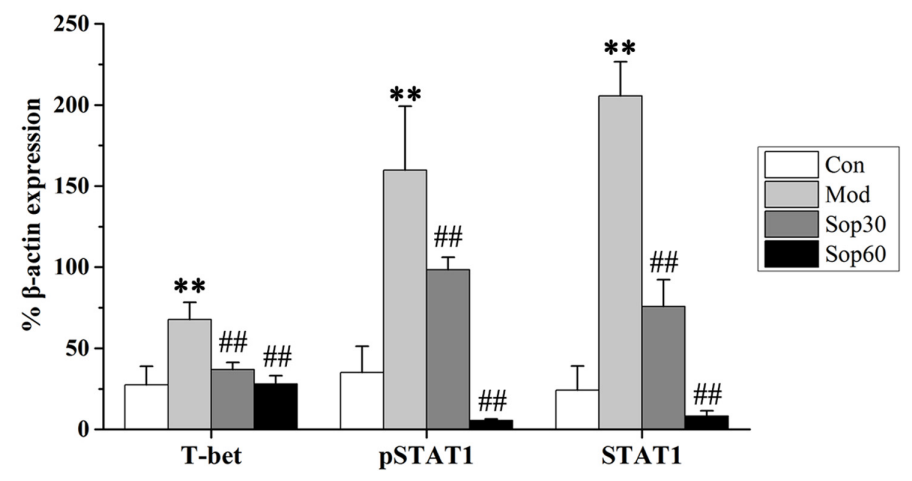

FIGURE 5 | Sophocaripine downregulated the expression of T-bet via inhibiting STAT1 activation and overexpression of SOCS1. Mice received Sophocaripine pretreatment and ConA injection as described. The mRNA expression levels of (A) SOCS1, (B) GATA3, (C) T-bet were measured by q-pcr ( $n=6$ each group). The protein expression of (D) T-bet, pSTAT1, STAT1 were measured by Western blot. (E) The data analysis of Western blot $(n=3)$. ${ }^{* *} p<0.01$ compared with the control group, ${ }^{\#} p<0.05,{ }^{\# \#} p<0.01$ compared with the model group.

and attenuate liver injury induced by Poly I: C/D-GalN and suppressed NK cell activation (Huang et al., 2016).

In the present study, we showed that Sophocarpine could protect against $\mathrm{T}$ cell-dependent immune hepatitis induced by ConA. Pretreatment with Sophocarpine significantly and dose-dependently downregulated the levels of ALT, AST, and TBIL. Histological staining significantly demonstrated that liver inflammation and apoptotic necrosis was dramatically reduced in Sophocarpine-pretreated mice. Sophocarpine also significantly reduced the release of chemokines and adhesion molecules as well as pro-inflammatory cytokines.

Con $\mathrm{A}$ is a well-known T-cell mitogen, after ConA administration, the levels of inflammatory cytokine is elevate subsequently resulting in abundant liver necrosis with mass lymphocytes infiltration (Kato et al., 2013). Chemokines and adhesion molecules play important roles in inflammatory and immune responses in ConA-induced hepatitis (Luo et al., 2015). Chemotactic factors was activated which attracted leukocytes into the liver to amplify the inflammatory response ( $\mathrm{Xu}$ et al., 2008). MIP-1 $\alpha$ has been shown to significantly contribute to the pathogenesis of ConA-induced hepatitis as hepatic injury was strikingly attenuated in mice with MIP-1 $\alpha$ deficiency (Ajuebor et al., 2004). In addition, CXCL10 has been reported to be a specific chemoattractant for $\mathrm{T}$ lymphocytes, and the expression of CXCL10 was specifically induced in the livers of mice treated with ConA (Tamaru et al., 2000). Furthermore, ICAM1 has been reported to contribute to the development of hepatitis by mediating adhesion and supporting the migration of lymphocytes into the liver (Kawasuji et al., 2006). In the present study, we found that Sophocarpine inhibited MIP-1 $\alpha$, CXCL10, and ICAM-1 mRNA expression, which in turn inhibited the infiltration of leukocytes, thereby alleviating the liver damage induced by ConA.

IFN- $\gamma$ has been reported to be a critical mediator of liver injury in the pathogenesis of ConA-induced hepatitis (Kusters et al., 1996). ConA-mediated liver damage may be prevented by blocking IFN- $\gamma$ (Tagawa et al., 1997). Furthermore, IFN- $\gamma$ is a major cytokine responsible for STAT1 activation. Disruption of the IFN- $\gamma$ or STAT1 genes abolished elevated ALT activities and necrosis, suggesting that IFN- $\gamma /$ STAT1 plays an essential role in ConA-induced hepatitis (Hong et al., 2002). In this signaling pathway, SOCS1 was considered as an important negative regulation of the cytokine-JAK-STAT pathway and forced expression of SOCS1 could prevented ConA-induced liver injury by suppressing STAT1 activation (Torisu et al., 2008; Yoshimura, 2009). Constitutive activation of STAT1 as well as constitutive expression of IFN- $\gamma$-inducible genes was observed in SOCS1 KO mice. Furthermore, T cell-specific SOCS1 
deficient mice demonstrated an enhanced sensitivity to ConAinduced hepatitis (Hashimoto et al., 2011; Yoshimura et al., 2012). In the current study, Sophocarpine pretreatment significantly inhibited STAT1 activity and increased the expression of SOCS1, subsequently downregulating its signaling proteins T-bet and inhibiting the production of Th1 cytokines, which protects mice from ConA-induced acute hepatitis. However, the precise mechanism underlying this process remains unknown.

\section{CONCLUSION}

In summary, we used the T cell-dependent hepatitis mouse model to examine the protective effects of Sophocarpine. In the present study, Sophocarpine attenuated ConA-induced liver injury in mice. The protective effect of Sophocarpine was associated with its inhibition of inflammatory mediators, chemokines, and the STAT1 signaling pathway. Taken together, the results of this study

\section{REFERENCES}

Ajuebor, M. N., Hogaboam, C. M., Le, T., Proudfoot, A. E., and Swain, M. G. (2004). CCL3/MIP- $1 \alpha$ is pro-inflammatory in murine T cell-mediated hepatitis by recruiting CCR1-expressing CD4+ T cells to the liver. Eur. J. Immunol. 34, 2907-2918. doi: 10.1002/eji.200425071

Ding, R. B., Tian, K., Huang, L. L., He, C. W., Jiang, Y., Wang, Y. T., et al. (2012). Herbal medicines for the prevention of alcoholic liver disease: a review. J. Ethnopharmacol. 144, 457-465. doi: 10.1016/j.jep.2012.09.044

Gao, Y., Jiang, W., Dong, C., Li, C., Fu, X., Min, L., et al. (2012). Anti-inflammatory effects of sophocarpine in LPS-induced RAW 264.7 cells via NF-кB and MAPKs signaling pathways. Toxicol. In Vitro 26, 1-6. doi: 10.1016/j.tiv.2011. 09.019

Gao, Y., Li, G., Li, C., Zhu, X., Li, M., Fu, C., et al. (2009). Anti-nociceptive and anti-inflammatory activity of sophocarpine. J. Ethnopharmacol. 125, 324-329. doi: 10.1016/j.jep.2009.06.036

Hashimoto, M., Ayada, T., Kinjyo, I., Hiwatashi, K., Yoshida, H., Okada, Y., et al. (2009). Silencing of SOCS1 in macrophages suppresses tumor development by enhancing antitumor inflammation. Cancer Sci. 100, 730-736. doi: 10.1111/j. 1349-7006.2009.01098.x

Hashimoto, M., Hiwatashi, K., Ichiyama, K., Morita, R., Sekiya, T., Kimura, A., et al. (2011). SOCS1 regulates type I/type II NKT cell balance by regulating IFN $\gamma$ signaling. Int. Immunol. 23, 165-176. doi: 10.1093/intimm/ dxq469

Hong, F., Jaruga, B., Kim, W. H., Radaeva, S., El-Assal, O. N., Tian, Z., et al. (2002). Opposing roles of STAT1 and STAT3 in T cell-mediated hepatitis: regulation by SOCS. J. Clin. Invest. 110, 1503-1513. doi: 10.1172/JCI15841

Hu, Y., Wang, S., Wu, X., Zhang, J., Chen, R., Chen, M., et al. (2013). Chinese herbal medicine-derived compounds for cancer therapy: a focus on hepatocellular carcinoma. J. Ethnopharmacol. 149, 601-612. doi: 10.1016/j.jep.2013. 07.030

Huang, Y. Q., Li, P. Y., Wang, J. B., Zhou, H. Q., Yang, Z. R., Yang, R. C., et al. (2016). Inhibition of sophocarpine on poly I: C/D-GalN-induced immunological liver injury in mice. Front. Pharmacol. 7:256. doi: 10.3389/fphar. 2016.00256

Jaruga, B., Hong, F., Kim, W.-H., and Gao, B. (2004). IFN- $\gamma /$ STAT1 acts as a proinflammatory signal in $\mathrm{T}$ cell-mediated hepatitis via induction of multiple chemokines and adhesion molecules: a critical role of IRF-1. Am. J. Physiol. Gastrointest. Liver Physiol. 287, G1044-G1052. doi: 10.1152/ajpgi.00184. 2004

Kato, J., Okamoto, T., Motoyama, H., Uchiyama, R., Kirchhofer, D., Van Rooijen, N., et al. (2013). Interferon-gamma-mediated tissue factor expression contributes to T-cell-mediated hepatitis through induction of hypercoagulation in mice. Hepatology 57, 362-372. doi: 10.1002/hep.26027 provide new opportunities for the use of Sophocarpine in the prevention and treatment of immune-mediated liver disease.

\section{AUTHOR CONTRIBUTIONS}

Z-FB, J-BW, and X-HX contributed to research design; X-XS performed the research; S-JL, H-HS, and R-LW provided innovative views and opinions; Y-MZ, Y-MG, and MN analyzed the data; and X-XS and C-EZ wrote the manuscript.

\section{ACKNOWLEDGMENTS}

This work was supported by the National Natural Science Foundation of China (no. 81330090) and the Chinese Medicine Antiviral Collaborative Innovation Center (no. XTCX2014 B01-06).

Kawasuji, A., Hasegawa, M., Horikawa, M., Fujita, T., Matsushita, Y., Matsushita, T., et al. (2006). L-selectin and intercellular adhesion molecule-1 regulate the development of Concanavalin A-induced liver injury. J. Leukoc. Biol. 79, 696-705. doi: 10.1189/jlb.0905527

Kusters, S., Gantner, F., Kunstle, G., and Tiegs, G. (1996). Interferon gamma plays a critical role in T cell-dependent liver injury in mice initiated by concanavalin A. Gastroenterology 111, 462-471. doi: 10.1053/gast.1996.v111.pm869 0213

Luo, Q., Zhu, L., Ding, J., Zhuang, X., Xu, L., and Chen, F. (2015). Protective effect of galangin in Concanavalin A-induced hepatitis in mice. Drug Des. Devel. Ther. 9, 2983-2992. doi: 10.2147/DDDT.S80979

Manns, M. P., Lohse, A. W., and Vergani, D. (2015). Autoimmune hepatitis-update 2015. J. Hepatol. 62, S100-S111. doi: 10.1016/j.jhep.2015.03.005

Miyagi, T., Takehara, T., Tatsumi, T., Suzuki, T., Jinushi, M., Kanazawa, Y., et al. (2004). Concanavalin a injection activates intrahepatic innate immune cells to provoke an antitumor effect in murine liver. Hepatology 40, 1190-1196. doi: 10.1002/hep.20447

Ogata, H., Chinen, T., Yoshida, T., Kinjyo, I., Takaesu, G., Shiraishi, H., et al. (2006). Loss of SOCS3 in the liver promotes fibrosis by enhancing STAT3mediated TGF-betal production. Oncogene 25, 2520-2530. doi: 10.1038/sj.onc. 1209281

Qian, H., Shi, J., Fan, T. T., Lv, J., Chen, S. W., Song, C. Y., et al. (2014). Sophocarpine attenuates liver fibrosis by inhibiting the TLR4 signaling pathway in rats. World J. Gastroenterol. 20, 1822-1832. doi: 10.3748/wjg.v20.i7.1822

Sahin, H., Borkham-Kamphorst, E., Berres, M. L., Kaldenbach, M., Schmitz, P., Weiskirchen, R., et al. (2013). Proapoptotic effects of the chemokine, CXCL 10 are mediated by the noncognate receptor TLR4 in hepatocytes. Hepatology 57, 797-805. doi: 10.1002/hep.26069

Schumann, J., Prockl, J., Kiemer, A. K., Vollmar, A. M., Bang, R., and Tiegs, G. (2003). Silibinin protects mice from T cell-dependent liver injury. J. Hepatol. 39, 333-340. doi: 10.1016/S0168-8278(03)00239-3

Shinohara, M. L., Jansson, M., Hwang, E. S., Werneck, M. B., Glimcher, L. H., and Cantor, H. (2005). T-bet-dependent expression of osteopontin contributes to T cell polarization. Proc. Natl. Acad. Sci. U.S.A. 102, 17101-17106. doi: 10.1073/pnas.0508666102

Siebler, J., Wirtz, S., Klein, S., Protschka, M., Blessing, M., Galle, P. R., et al. (2003). A key pathogenic role for the STAT1/T-bet signaling pathway in T-cellmediated liver inflammation. Hepatology 38, 1573-1580. doi: 10.1016/j.hep. 2003.09.020

Stummvoll, G. H., DiPaolo, R. J., Huter, E. N., Davidson, T. S., Glass, D., Ward, J. M., et al. (2008). Th1, Th2, and Th17 effector T cell-induced autoimmune gastritis differs in pathological pattern and in susceptibility to suppression by regulatory T cells. J. Immunol. 181, 1908-1916. doi: 10.4049/jimmunol.181.3. 1908 
Tagawa, Y., Sekikawa, K., and Iwakura, Y. (1997). Suppression of concanavalin A-induced hepatitis in IFN-gamma(-/-) mice, but not in TNF-alpha(-/-) mice: role for IFN-gamma in activating apoptosis of hepatocytes. J. Immunol. 159, 1418-1428.

Tamaru, M., Nishioji, K., Kobayashi, Y., Watanabe, Y., Itoh, Y., Okanoue, T., et al. (2000). Liver-infiltrating $\mathrm{T}$ lymphocytes are attracted selectively by IFNinducible protein-10. Cytokine 12, 299-308. doi: 10.1006/cyto.1999.0560

Torisu, T., Nakaya, M., Watanabe, S., Hashimoto, M., Yoshida, H., Chinen, T., et al. (2008). Suppressor of cytokine signaling 1 protects mice against concanavalin A-induced hepatitis by inhibiting apoptosis. Hepatology 47, 1644-1654. doi: 10.1002/hep.22214

Wang, H. X., Liu, M., Weng, S. Y., Li, J. J., Xie, C., He, H. L., et al. (2012). Immune mechanisms of Concanavalin A model of autoimmune hepatitis. World J. Gastroenterol. 18, 119-125. doi: 10.3748/wjg.v18.i2.119

Xu, Y., Feng, D., Wang, Y., Lin, S., and Xu, L. (2008). Sodium tanshinone IIA sulfonate protects mice from ConA-induced hepatitis via inhibiting NF-kappaB and IFN-gamma/STAT1 pathways. J. Clin. Immunol. 28, 512-519. doi: 10.1007/ s10875-008-9206-3

Yoshimura, A. (2009). Regulation of cytokine signaling by the SOCS and Spred family proteins. Keio J. Med. 58, 73-83. doi: 10.2302/kjm.58.73

Yoshimura, A., Suzuki, M., Sakaguchi, R., Hanada, T., and Yasukawa, H. (2012). SOCS, inflammation, and autoimmunity. Front. Immunol. 3:20. doi: 10.3389/ fimmu.2012.00020
Yuan, H., Ma, Q., Ye, L., and Piao, G. (2016). The traditional medicine and modern medicine from natural products. Molecules 21:E559. doi: 10.3390/ molecules21050559

Zhang, J., Wang, W., and Duan, Z. (2007). Progress of research and application of matrine-type alkaloids. Prog. Mod. Biomed. 7, 451-455.

Zheng, W., Wang, Q., Lu, X., Shi, Q., Zou, J., Tao, Y., et al. (2016). Protective effects of Dracocephalum heterophyllum in ConA-induced acute hepatitis. Mediators Inflamm. 2016:2684321. doi: 10.1155/2016/2684321

Zhu, J., Yamane, H., and Paul, W. E. (2010). Differentiation of effector CD4 T cell populations $\left(^{*}\right)$. Annu. Rev. Immunol. 28, 445-489. doi: 10.1146/annurevimmunol-030409-101212

Conflict of Interest Statement: The authors declare that the research was conducted in the absence of any commercial or financial relationships that could be construed as a potential conflict of interest.

Copyright (C) 2017 Sang, Wang, Zhang, Liu, Shen, Guo, Zhang, Niu, Wang, Bai and Xiao. This is an open-access article distributed under the terms of the Creative Commons Attribution License (CC BY). The use, distribution or reproduction in other forums is permitted, provided the original author(s) or licensor are credited and that the original publication in this journal is cited, in accordance with accepted academic practice. No use, distribution or reproduction is permitted which does not comply with these terms. 